\title{
Energy-Efficient Resource Allocation for 6G Backscatter-Enabled NOMA IoV Networks
}

\author{
Wali Ullah Khan, Muhammad Awais Javed, Tu N. Nguyen, Shafiullah Khan, Basem M. Elhalawany
}

\begin{abstract}
The integration of Ambient Backscatter Communication (AmBC) with Non-Orthogonal Multiple Access (NOMA) is expected to support connectivity of low-powered Internetof-Vehicle (IoV) in the upcoming Sixth-Generation (6G) transportation system. This paper proposes an energy-efficient resource allocation framework for the AmBC-enabled NOMA IoV network under imperfect Successive Interference Cancellation (SIC) decoding. In particular, multiple Road-Side Units (RSUs) transmit superimposed signals to their associated IoVs utilizing downlink NOMA transmission. Meanwhile, the Backscatter Tags (BackTags) also transmit data symbols towards nearby IoVs by reflecting the superimposed signals of RSUs. Thus, the objective is to maximize the total energy efficiency of the NOMA IoV network subject to the minimum data rate of all IoVs. A joint problem that simultaneously optimizes the total power budget of each RSU, power allocation coefficient of IoVs and reflection power of BackTags under imperfect SIC decoding is formulated. A Dinkelbach approach is first adopted to transform the optimization problem and then the transformed problem is decoupled into two subproblems for optimal transmit power at RSUs and efficient reflection power at BackTags, respectively. To solve the problems efficiently, dual theory and KarushKuhn-Tucker conditions are exploited, where the Lagrangian dual variables are iteratively calculated using the subgradient method. To check the performance of the proposed framework, a benchmark optimization without backscattering is also provided. Numerical results demonstrate the superiority of the proposed AmBC-enabled NOMA IoV framework over the benchmark conventional IoV framework.
\end{abstract}

Index Terms-6G, ambient backscatter communication (AmBC), non-orthogonal multiple access (NOMA), imperfect successive interference cancellation (SIC), Dinkelbach method.

\section{INTRODUCTION}

$\mathbf{I}$ NTELLIGENT Transportation System (ITS) is a key component of future smart cities [1]. Using robust wireless communications and advanced sensors, future transportation will be revolutionized in terms of safety and comfort [2]. Many applications such as safety awareness, autonomous driving, road traffic management, and infotainment are offered by future ITS [3]. The future sixth-generation $(6 \mathrm{G})$ will be the

Wali Ullah Khan is with the Interdisciplinary Center for Security, Reliability and Trust (SnT)/ SigCom, University of Luxembourg, 4365 Esch-sur-Alzette, Luxembourg (e-mail: waliullah.khan@uni.lu).

Muhammad Awais Javed is with the Department of Electrical and Computer Engineering, COMSATS University Islamabad, Islamabad 45550, Pakistan. (email: awais.javed@comsats.edu.pk).

Tu N. Nguyen is with the Department of Computer Science, Kennesaw State University, Marietta, GA 30060, USA (email: tu.nguyen@kennesaw.edu).

Shafiullah Khan is with Department of Electronics, Islamia College University, Peshawar, Pakistan (email: shafielectronics@icp.edu.pk).

Basem M. Elhalawany is with the College of Computer Science, Shenzhen University, China, and with Benha University, Egypt (email: basem.mamdoh@szu.edu.cn, basem.mamdoh@feng.bu.edu.eg).

Corresponding author: Tu N. Nguyen core wireless technology used for future ITS that will provide ubiquitous connectivity, secure data sharing, energy-efficient transmissions, and rapid computation [4]. As compared to fifth-generation (5G) enabled Internet of Vehicles (IoVs) that focuses on autonomous driving, $6 \mathrm{G}$ will be driven by ensuring reliability in the autonomous driving, sharing of more detailed road traffic information, Augmented Reality (AR) and Virtual Reality (VR) based traffic planning, and advanced multimedia and gaming applications [5]. 6G enabled IoVs will provide extremely high data rates of the order of Tera-bits per second. It will also enhance the packet delivery ratio to $99.99999 \%$ by and reduce latency to less than $1 \mathrm{~ms}$, thus increasing the reliability of wireless communications by many folds [6]. Many new technologies such as reconfigurable intelligent surfaces [7], terahertz communications [8], blockchain technology [9], ambient backscatter communications (AmBC) [10], Non-orthogonal Multiple Access (NOMA) [11], and federated learning [12] will be the major enablers of reliable communications offered by $6 \mathrm{G}$.

The major challenges for connecting massive IoVs in $6 \mathrm{G}$ would be the limited energy reservoirs and spectrum resources. In this regard, AmBC and NOMA are the two promising candidate technologies [13], [14]. Utilizing the existing Radio-frequency (RF) signal, AmBC will allow IoV and roadside-unites (RSU) to operate with little battery power, hence providing considerable energy savings and reducing carbon footprints. The basic principle of AmBC is to allow a backscatter tag (BackTag) to transmit data by reflecting and modulating the existing RF signal. Moreover, NOMA will be extensively used in the $6 \mathrm{G}$ enabled IoV network for radio access due to its ability to accommodate multiple IoVs over the same spectrum/time resources. NOMA first uses superposition coding at the transmitter side to encode multiple signals over the same spectrum using power multiplexing and then exploits successive interference cancellation (SIC) at the receiver side to decode these signals [15]. In the last several years, a lot of works have been done on traditional NOMA networks [16]-[19]. Recently, researchers have shown interest in the integration of AmBC and NOMA with vehicular networks for providing energy-efficient and reliable communications.

\section{A. Related Works}

Researches from industry and academia have studied various problems on NOMA vehicular networks. For instance, Reference [20] has provided hyper-graph-based resource allocation to maximize the total achievable capacity of NOMA vehicular networks using device to device (D2D) communications. Abbasi et al. [21] have proposed the unmanned aerial vehicle-based cooperative power allocation framework 
to maximize the sum rate of the system. In [22], Zheng et al. have optimized resource allocation to improve the maxmin fairness of NOMA vehicular networks. The work of [23] has designed a decentralized algorithm and derived a closed-form solution to investigate the exact capacity of both urban and crowded vehicular networks. Xiao et al. [24] have proposed efficient vehicle clustering and optimal power control to maximize the achievable rate of the system. The authors of [25] have designed an efficient iterative distribution-based algorithm to reduce the latency and improve the reliability of NOMA vehicular networks. In a similar study, Guo et al. [26] have optimized frequency and power allocation to improve the reliability of the NOMA system. Khan et al. [27] have proposed an efficient power management scheme to investigate the trade-off between energy and spectrum efficiency of NOMA vehicular networks. The work in [28] has solved an energy efficiency optimization problem in NOMA vehicular networks using the Dinkelbach approach. Do et al. [29] computed the exact and closed-form solutions to maximize the ergodic capacity of full-duplex NOMA vehicular networks using D2D communications.

Recently, the integration of NOMA in AmBC has triggered an overwhelming research interest due to energy and spectral efficient communications. For example, the work in [30] has derived the closed-form solution for the outage probability and ergodic capacity of NOMA AmBC systems. The works in [31], [32] have investigated the problems of physical layer security to maximize the secrecy rate of AmBC under multiple eavesdroppers. In [33], [34], Li et al. have calculated outage and intercept probabilities to study the security and reliability of AmBC under i) in-phase and quadrature-phase imbalance and ii) imperfect SIC, channel state information (CSI), and residual hardware impairment. By using the efficient selection criterion of reflection coefficient, Guo et al. [35] have improved the average successful decoding bit of AmBC systems. The researchers in [36], [37] have explored the joint optimization problems of transmit power and reflection coefficient to maximize the sum capacity of NOMA AmBC networks under perfect and imperfect SIC decoding. Liao et al. [38] have jointly optimized the transmit power and subcarrier at the access point, time allocation, and reflection coefficient at BackTags to enhance the minimum throughput of the AmBC systems. Similarly, the research work in [39] has optimized the time allocation and reflection coefficient to maximize the minimum throughput of the NOMA systems. Xu et al. [40] have provided a joint optimization framework of transmit power and reflection coefficient to maximize the energy efficiency of NOMA AmBC networks. Moreover, researchers have also studied different problems in AmBC using artificial intelligence techniques [41], [42]. The researches of [43] have also proposed the optimization of reflection coefficient to maximize the secrecy rate of NOMA AmBC networks. Of late Khan et al. [44] have investigated spectral efficiency of multi-cell NOMA AmBC vehicular networks.

\section{B. Motivation and Contributions}

The researches in [20]-[29] have only studied NOMA and vehicular communications with perfect SIC assumptions.
Moreover, they do not consider AmBC in their system models. Besides that, the authors of [30]-[44] have studied AmBC but they do not consider vehicular communications. Although the works in [34], [44] have considered imperfect SIC decoding but their system models, objectives and approaches are different. Also, most of the above literature considers singlecell scenarios. To the best of our knowledge, the optimization framework that simultaneously optimizes the total power budget of each RSU, power allocation coefficient of IoVs, and reflection coefficient of BackTags to maximize the total achievable energy efficiency of multi-RSU NOMA AmBC network has not yet been investigated. To fill this research gap, we aim to propose a new optimization to maximize the total energy efficiency of the system under imperfect SIC decoding. We first employ the Dinkelbach method to transform the objective function of the original problem into a subtractive function. Since the optimization problem is coupled on different variables and it makes it con-convex and difficult to solve. Thus, we decouple it into two subproblems for transmit power at RSU and reflection coefficient of BackTag in each cell. Then, we exploit dual theory and KKT conditions to calculate the closed-form solutions. The simulation results are compared with the benchmark NOMA optimization framework without AmBC which shows the superiority in terms of total achievable energy efficiency.

The main contribution of our paper can be summarized as follow:

- We consider a realistic $6 G$ vehicular scenario where multiple RSUs communicate with their associated IoVs through downlink NOMA protocol. The BackTags also transmit signals toward near IoVs by reflecting and modulating the RF signals of the RSUs.

- We optimize three variables including, the total power budget of each RSUs, the power allocation coefficient of IoVs for NOMA transmissions, and the reflection power of BackTags. Thus, the objective is to maximize the total energy efficiency of the AmBC network under imperfect SIC decoding.

- We use the Dinkelbach method to transform the objective function of the non-convex energy efficiency maximization problem into a subtractive one. Then we decouple the problem into two subproblems for transmit power allocation and reflection power selection. Subsequently, we adopt dual theory and KKT conditions to derive closed-form solutions.

- Numerical results highlight the significance of the proposed optimization framework as compared to the conventional NOMA IoV network without AmBC. Moreover, the proposed optimization framework exhibits fast convergence and low complexity.

The rest of our paper is structured as follows: System model and problem formulation are provided in Section II. The proposed resource allocation solution is discussed in Section III. Numerical results and discussion are presented in Section IV. Concluding remarks are provided in Section V. 


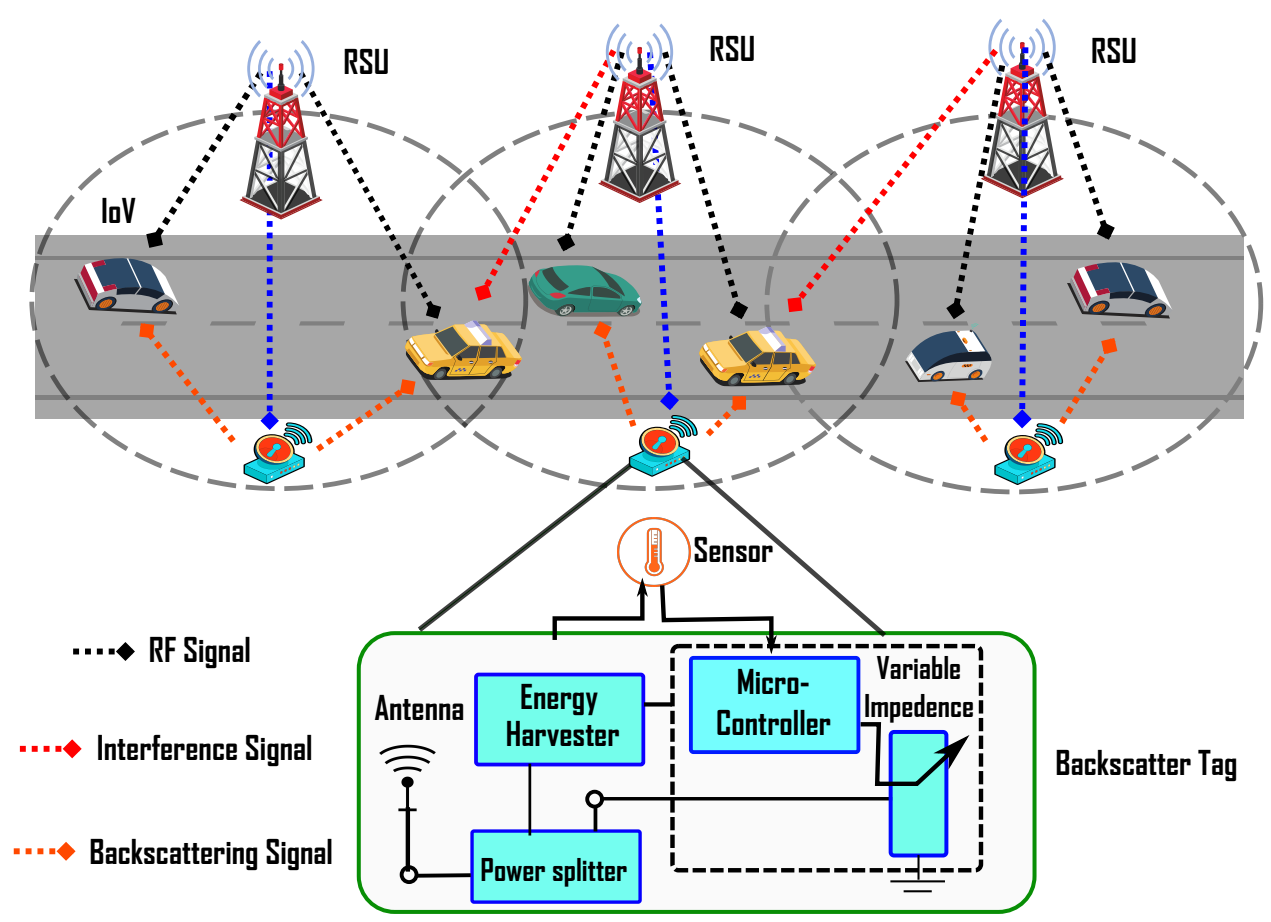

Fig. 1: System model

\section{System Model AND PROBlem Formulation}

As shown in Fig. 1, the AmBC-enabled vehicular network is considered, where $M$ RSUs communicate with $\Pi / 2$ vehicle pairs utilizing NOMA protocol. The term $\Pi$ is the number of vehicles such that $\Pi=2 M$. Each pair consists of two IoVs, one with good channel conditions (stated as $s$ IoV) and another with weak channel conditions (denoted as $w$ IoV), respectively. The system also composes of $K$ BackTags, where they receive the superimposed signals of RSUs, harvest the radio frequency energy to operate the circuits, modulate information, and reflect it towards nearby IoVs. It is assumed that: 1) RSUs, BackTags, and IoVs are equipped with omnidirectional antenna; 2) RSUs share the same spectrum at the same time; 3) The channels are independent and identical Rayleigh fading [45]; 4) Due to the error propagation in SIC decoding, IoVs can not always perfectly decode their signals; 5) The channel state information are known in the system because we do not consider the mobility of IoVs due to rapid changes in their channel characteristics [46]. By considering the mobility in IoVs, various admission control and IoVs association policies would be required. A superimposed signal of $m \in M \mathrm{RSU}$ for its associated IoVs pair is written as $x_{m}=\sqrt{P_{m} \varpi_{s, m}} x_{s, m}+\sqrt{P_{m} \varpi_{w, m}} x_{w, m}$, where $P_{m}$ is the allocated power of $m \mathrm{RSU}, \varpi_{s, m}$ and $\varpi_{w, m}$ are the power allocation coefficient of $m$ RSU for $s$ IoV and $w$ IoV, respectively. Furthermore, $x_{s, m}$ and $x_{w, m}$ denote the data symbols of $s$ IoV and $w$ IoV. Meanwhile, $k$ BackTag also receives $x_{m}$ from $m$ RSU, reflect it towards $s$ IoV and $w$ IoV by modulating own symbol $z(t)$ such as $\mathbb{E}\left[|z(t)|^{2}\right]=1$, where $\mathbb{E}[$.$] shows the expectation operation. The signals that$ $s$ IoV and $w$ IoV receive from $m$ RSU and $k$ BackTag can be expressed as

$$
\begin{aligned}
y_{s, m} & \left.=\sqrt{h_{s, m}} x_{m}+\sqrt{\psi_{k, m} g_{s, k}^{m}\left(h_{k, m}\right.} x_{m}\right) z(t) \\
& +\sum_{m^{\prime}=1, m^{\prime} \neq m}^{M} \sqrt{P_{m^{\prime}} h_{s, m^{\prime}}^{m}} x_{m^{\prime}}+\xi_{s, m}, \\
y_{w, m} & \left.=\sqrt{h_{w, m}} x_{m}+\sqrt{\psi_{k, m} g_{w, k}^{m}\left(h_{w, k}^{m}\right.} x_{m}\right) z(t) \\
& +\sum_{m^{\prime}=1, m^{\prime} \neq m}^{M} \sqrt{P_{m^{\prime}} h_{w, m^{\prime}}^{m}} x_{m^{\prime}}+\xi_{w, m},
\end{aligned}
$$

where the first terms in both equations are the signals from $m$ $\mathrm{RSU}$, the second terms are reflected signals from the $k$ Backag, the third terms denote the interference from the nearby RSUs, and the last terms are white Gaussian noise having $\sigma^{2}$ variance. Besides, $h_{s, m}$ and $h_{w, m}$ represent the channel gains of $s$ IoV and $w$ IoV from $m$ RSU, $h_{k, m}$ is the channel gain of $k$ BackTag from the $m \mathrm{RSU}$, and $\psi_{k, m}$ denotes the reflection power of $k$ BackTag. Moreover, $g_{s, k}^{m}$ and $g_{w, k}^{m}$ are the channel gains from $k$ BackTag to $s \mathrm{IoV}$ and $w$ IoV, respectively. In addition, $P_{m^{\prime}}$ denotes the interference power from $m^{\prime} \mathrm{RSU}$, $h_{s, m^{\prime}}^{m}$ and $h_{w, m^{\prime}}^{m}$ represent the interference channel gains from $m^{\prime}$ RSU to $s$ IoV and $w$ IoV. Based on the NOMA protocol, $s$ IoV can decode its signal from RSU and BackTag by applying SIC decoding technique. However, $w$ IoV cannot apply SIC and decodes its signal by treating other signals as noise. As discussed earlier, IoVs cannot always decode perfect signals. The signal to interference plus noise ratio (SINR) of $s$ IoV to decode the signal of $w$ IoV and its own and the SINR of $w$ IoV to decode its signal can be written as

$$
\gamma_{s \rightarrow w}^{m}=\frac{P_{m} \varpi_{w, m}\left|h_{s, m}\right|^{2}+H_{s, w}}{P_{m} \varpi_{s, m}\left(\left|h_{s, m}\right|^{2}+H_{s, w}\right)+I_{w, m^{\prime}}^{m}+\sigma^{2}},
$$




$$
\begin{gathered}
\gamma_{s \rightarrow s}^{m}=\frac{P_{m} \varpi_{s, m}\left(\left|h_{s, m}\right|^{2}+G_{s, m}\right)}{P_{m} \varpi_{w, m}\left|h_{s, m}\right|^{2} \Lambda+I_{s, m^{\prime}}^{m}+\sigma^{2}}, \\
\gamma_{w \rightarrow w}^{m}=\frac{P_{m} \varpi_{w, m}\left(\left|h_{w, m}\right|^{2}+G_{w, m}\right)}{P_{m} \varpi_{s, m}\left(\left|h_{w, m}\right|^{2}+G_{w, m}\right)+I_{w, m^{\prime}}^{m}+\sigma^{2}},
\end{gathered}
$$

$H_{s, w}=\psi_{k, m}\left|h_{k, m}\right|^{2}\left|g_{s, k}^{m}\right|^{2}, I_{w, m^{\prime}}^{m}=\left|h_{w, m^{\prime}}^{m}\right|^{2} \sum_{m^{\prime}=1}^{M} P_{m^{\prime}}$, $G_{s, m}=\psi_{k, m}\left|h_{k, m}\right|^{2}\left|g_{s, k}^{m}\right|^{2}, \quad G_{w, m}=\psi_{k, m}\left|h_{k, m}\right|^{2}\left|g_{w, k}^{m}\right|^{2}$, and $\Lambda$ is the imperfect SIC parameter which is given as $\Lambda=\mathbb{E}\left[\left|z_{s, m}-\tilde{z}_{s, m}\right|^{2}\right]$, where $z_{s, m}-\tilde{z}_{s, m}$ denotes the difference between the original signal and the estimated signal. Based on the above SINR expressions, the data rate of $s$ IoV and $w$ IoV can be written as $R_{s, m}=\log _{2}\left(1+\gamma_{s \rightarrow s}^{m}\right)$ and $R_{w, m}=\log _{2}\left(1+\gamma_{w \rightarrow w}^{m}\right)$, respectively.

In this work, we seek to maximize the total energy efficiency of AmBC-enabled NOMA IoV network. The total energy efficiency of IoV network can be defined as

$$
\eta_{E E}\left(\varpi_{k}, P_{m}, \psi_{k, m}\right)=\sum_{m=1}^{M} \frac{R_{m}\left(\varpi_{k}, P_{m}, \psi_{k, m}\right)}{P_{t o t}\left(\varpi_{k}, P_{m}, \psi_{k, m}\right)},
$$

where $\varpi_{k}=\left\{\varpi_{s, m}, \varpi_{w, m}\right\}$ is the power allocation coefficient, $R_{m}=R_{s, m}+R_{w, m}$ denotes the total achievable data rate of $m \mathrm{RSU}$, and $P_{t o t}=P_{m}\left(\Lambda_{s, m}+\Lambda_{w, m}\right)+P_{c}$ its total power consumption, where $P_{c}$ is the circuit power. This can be achieved by jointly optimizing the power budget of RSU, power allocation coefficient of IoVs, and reflection power of BackTag in each RSU under imperfect SIC decoding. Moreover, the minimum data rate of individual IoV is also guaranteed. This problem which simultaneously optimizes all the above variables can be formulated as

$$
\begin{array}{ll}
\text { (P) } & \underset{\left(\varpi_{k}, P_{m}, \psi_{k}, m\right.}{\operatorname{maximize}} \eta_{E E}\left(\varpi_{k}, P_{m}, \psi_{k, m}\right) \\
\text { s.t. } & P_{m} \varpi_{s, m}\left(\left|h_{s, m}\right|^{2}+G_{s, m}\right) \geq\left(2^{R_{m i n}}-1\right) \\
& \left(\left|h_{s, m}\right|^{2} P_{m} \varpi_{w, m} \Lambda+I_{s, m^{\prime}}^{m}+\sigma^{2}\right), \forall m, \\
& P_{m} \Lambda_{w, m}\left(\left|h_{w, m}\right|^{2}+G_{w, m}\right) \geq\left(2^{R_{m i n}}-1\right) \\
& \left(P_{m} \varpi_{s, m}\left(\left|h_{w, m}\right|^{2}+G_{w, m}\right)+I_{w, m^{\prime}}^{m}+\sigma^{2}\right), \forall m, \\
& P_{m} \varpi_{s, m} \leq P_{m} \varpi_{w, m}, \forall m, \forall s, w, \\
& 0 \leq P_{m} \leq P_{m a x}, \forall m, \\
& \varpi_{s, m}+\varpi_{w, m} \leq 1, \forall m, \\
& 0 \leq \psi_{k, m} \leq 1, \forall k, \forall m,
\end{array}
$$

where the objective in (7) is to maximize the total energy efficiency AmBC-enabled NOMA IoV network. Constraints (7a) and (7b) satisfy the minimum rate of $s$ IoV and $w$ IoV associated with $m$ RSU. Constraint (7c) ensures the power difference between $s$ IoV and $w$ IoV for efficient SIC decoding. Constraint (7d) limits the transmit power of each RSU while (7e) controls the allocation coefficients according to the NOMA protocol. In addition, constraint (7f) keeps the reflection power of BackTag between zero and one.

\section{Proposed Resource Allocation Solutions}

It can be observed that the above optimization problem in (7) is coupled on two variables; i) Power budgets of RSUs and power allocation coefficients of IoVs associated with each RSU; ii) Reflection power of BackTag located in the geographical area of each RSU. It makes the problem (P) very hard to solve. Thus, we transform the original problem by exploiting the Dinkelbach method. Then, for a given reflection power of BackTags, we first calculate the efficient power allocation coefficient of IoVs, and the power budget at each RSU. Then, we substitute the optimal values of power allocation coefficients and power budget into the original problem and compute the efficient reflection power of BackTags. Utilizing the Dinkelback method, we transform problem (7) as

$$
\begin{aligned}
& \text { (P1) } \underset{\left(\varpi_{k}, P_{m}, \psi_{k, m}\right)}{\operatorname{maximize}} \sum_{m=1}^{M} R_{m}\left(\varpi_{k}, P_{m}, \psi_{k, m}\right) \\
& -\beta \sum_{m=1}^{M} P_{t o t}\left(\varpi_{k}, P_{m}, \psi_{k, m}\right), \\
& \text { s.t. } \quad(7 a)-(7 f),
\end{aligned}
$$

where $\beta$ is the maximum energy efficiency which can be achieved as

$$
\begin{aligned}
& \sum_{m=1}^{M} R_{m}\left(\varpi_{\boldsymbol{k}}^{*}, P_{m}^{*}, \psi_{k, m}^{*}\right)- \\
& \beta^{*} \sum_{m=1}^{M} P_{t o t}\left(\varpi_{\boldsymbol{k}}^{*}, P_{m}^{*}, \psi_{k, m}^{*}\right)=0 .
\end{aligned}
$$

Next, we decouple the problem (8) into two subproblems for transmit power at RSUs and reflection power at BackTags.

\section{A. Efficient Transmit Power of RSUs}

To compute the efficient power allocation coefficient and transmit power budget at RSUs, we consider that the reflection power is given at BackTags. The optimization problem in (8) can be simplified as

$$
\begin{aligned}
& \text { (P2) } \underset{\left(\varpi_{m}, P_{m}\right)}{\operatorname{maximize}} \sum_{m=1}^{M} R_{m}\left(\varpi_{m}, P_{m}\right) \\
& -\beta \sum_{m=1}^{M} P_{\text {tot }}\left(\varpi_{m}, P_{m}\right), \\
& \text { s.t. } \quad(7 a)-(7 e),
\end{aligned}
$$

We can also write (4) and (5) as

$$
\gamma_{s \rightarrow s}^{m}=\frac{P_{m} \varpi_{s, m} \mathcal{A}_{s, m}}{P_{m} \varpi_{w, m} \mathcal{B}_{s, m}+\mathcal{C}_{s, m}},
$$

where $\mathcal{A}_{s, m}=\left|h_{s, m}\right|^{2}+G_{s, m}, \mathcal{B}_{s, m}=\left|h_{s, m}\right|^{2} \Lambda, \mathcal{C}_{s, m}=$ $I_{s, m^{\prime}}^{m}+\sigma^{2}$, and

$$
\gamma_{w \rightarrow w}^{m}=\frac{P_{m} \varpi_{w, m} \mathcal{A}_{w, m}}{P_{m} \varpi_{s, m} \mathcal{B}_{w, m}+\mathcal{C}_{w, m}},
$$

where $\mathcal{A}_{w, m}=\left|h_{w, m}\right|^{2}+G_{w, m}, \mathcal{B}_{w, m}=\left|h_{w, m}\right|^{2}+G_{w, m}$, $\mathcal{C}_{w, m}=I_{w, m^{\prime}}^{m}+\sigma^{2}$. Next we prove that the $R_{s, m}+R_{w, m}$ in (P2) is concave with respect to power allocation coefficients. 


\section{Proposition 1.}

$$
\begin{aligned}
& \log _{2}\left(1+\frac{P_{m} \varpi_{s, m} \mathcal{A}_{s, m}}{P_{m} \varpi_{w, m} \mathcal{B}_{s, m}+\mathcal{C}_{s, m}}\right) \\
& +\log _{2}\left(1+\frac{P_{m} \varpi_{w, m} \mathcal{A}_{w, m}}{P_{m} \varpi_{s, m} \mathcal{B}_{w, m}+\mathcal{C}_{w, m}}\right),
\end{aligned}
$$

is a concave function with respect to $\varpi_{m}=\left\{\varpi_{s, m}, \varpi_{w, m}\right\}$.

Proof. Please see Appendix A.

According to Proposition 1, the objective function of (P2) is concave-convex, which can be easily solved by dual theory. The dual problem associated with $(\mathrm{P} 2)$ can be defined as

$$
\underset{\boldsymbol{\lambda}_{\boldsymbol{m}}, \mu_{m}, \epsilon_{m} \geq 0}{\operatorname{minimize}} D\left(\boldsymbol{\lambda}_{\boldsymbol{m}}, \mu_{m}, \epsilon_{m}\right)
$$

where $\boldsymbol{\lambda}_{\boldsymbol{m}}=\left\{\lambda_{s, m}, \lambda_{w, m}\right\}, \mu_{m}, \epsilon_{m}$ are the dual variables. Now we define the dual function as

$$
D(.)=\underset{\varpi_{m}, P_{m}, \boldsymbol{\lambda}_{\boldsymbol{m}}, \mu_{m}, \epsilon_{m} \geq 0}{\operatorname{maximize}} \mathcal{L}\left(\varpi_{\boldsymbol{m}}, P_{m}, \boldsymbol{\lambda}_{\boldsymbol{m}}, \mu_{m}, \epsilon_{m}\right)
$$

where $\mathcal{L}($.$) is the Lagrangian function which can be derived$ as

$$
\begin{aligned}
& \mathcal{L}(.)=\sum_{m=1}^{M}\left\{\log _{2}\left(1+\frac{P_{m} \varpi_{s, m} \mathcal{A}_{s, m}}{P_{m} \varpi_{w, m} \mathcal{B}_{s, m}+\mathcal{C}_{s, m}}\right)\right. \\
& \left.+\log _{2}\left(1+\frac{P_{m} \varpi_{w, m} \mathcal{A}_{w, m}}{P_{m} \varpi_{s, m} \mathcal{B}_{w, m}+\mathcal{C}_{w, m}}\right)\right\} \\
& -\beta \sum_{m=1}^{M} P_{m}\left(\varpi_{s, m}+\varpi_{w, m}\right)+P_{c}+\lambda_{s, m}\left(P_{m} \varpi_{s, m} \mathcal{A}_{s, m}\right. \\
& \left.-\left(2^{R_{m i n}}-1\right) P_{m} \varpi_{w, m} \mathcal{B}_{s, m}+\mathcal{C}_{s, m}\right)+\lambda_{w, m}\left(P_{m} \varpi_{w, m}\right. \\
& \mathcal{A}_{w, m}-\left(2^{R_{m i n}}-1\right)\left(P_{m} \varpi_{s, m} \mathcal{B}_{w, m}+\mathcal{C}_{w, m}\right) \\
& +\mu_{m}\left(P_{\max }-P_{m}\right)+\epsilon_{m}\left(1-\varpi_{s, m}-\varpi_{w, m}\right)
\end{aligned}
$$

Next, we adopt KKT conditions, where the closed-form expressions of $s \mathrm{IoV}$ and $w \mathrm{IoV}$ can be expressed as

$$
\begin{aligned}
& \varpi_{s, m}^{*}=\left[\frac{-\Psi \pm \sqrt{\Psi^{2}-4 \Phi \Upsilon}}{2 \Phi}\right]^{+} \\
& \varpi_{w, m}^{*}=1-\varpi_{s, m}^{*}
\end{aligned}
$$

where $[.]^{+}=\max [0,$.$] and the values of$ $\Phi=P_{m}^{2}\left(-\mathcal{A}_{s, m} \mathcal{A}_{w, m} \mathcal{B}_{w, m}\left(1+\lambda_{s, m}\right)\left(\mathcal{C}_{s, m}+\right.\right.$ $\left.\mathcal{B}_{s, m} P_{m}\right)+\mathcal{A}_{s, m} \mathcal{B}_{w, m}^{2}\left(1+\lambda_{s, m}\right)\left(\mathcal{C}_{s, m}+\mathcal{B}_{s, m} P_{m}\right)+$ $\mathcal{A}_{s, m} \mathcal{A}_{w, m} \mathcal{B}_{s, m}\left(1+\lambda_{w, m}\right)\left(\mathcal{C}_{w, m}+\mathcal{B}_{w, m} P_{m}\right)-$ $\left.\mathcal{A}_{w, m} \mathcal{B}_{s, m}^{2}\left(1+\lambda_{w, m}\right)\left(\mathcal{C}_{w, m}+\mathcal{B}_{w, m} P_{m}\right)\right), \quad \Psi=$ $P_{m}\left(\mathcal{C}_{s, m}+\mathcal{B}_{s, m} P_{m}\right)\left(-\mathcal{A}_{s, m} \mathcal{C}_{w, m}\left(-2 \mathcal{B}_{w, m}\left(1+\lambda_{s, m}\right)+\right.\right.$ $\left.\mathcal{A}_{w, m}\left(2+\lambda_{w, m}\right)\right)+\mathcal{A}_{s, m} \mathcal{A}_{w, m} \mathcal{B}_{w, m}\left(\lambda_{s, m}-\lambda_{w, m}\right) P_{m}+$ $\left.2 \mathcal{A}_{w, m} \mathcal{B}_{s, m}\left(1+\lambda_{w, m}\right)\left(\mathcal{C}_{w, m}+\mathcal{B}_{w, m} P_{m}\right)\right), \Upsilon=$ $\left(\mathcal{C}_{s, m}+\mathcal{B}_{s, m} P_{m}\right)\left(\mathcal{A}_{s, m} \mathcal{C}_{w, m}^{2}\left(1+\lambda_{s, m}\right)+\mathcal{A}_{w, m}\left(-\mathcal{C}_{s, m}(1+\right.\right.$ $\left.\lambda_{w, m}\right)\left(\mathcal{C}_{w, m}+\mathcal{B}_{w, m} P_{m}\right)+P_{m}\left(\mathcal{A}_{s, m} \mathcal{C}_{w, m}\left(1+\lambda_{s, m}\right)-\right.$ $\left.\left.\left.\mathcal{B}_{s, m}\left(1+\lambda_{s, m}\right)\left(\mathcal{C}_{w, m}+\mathcal{B}_{w, m} P_{m}\right)\right)\right)\right)$,

Proof. Please see Appendix B.

Next we find the optimal power budget of each RSU. It can be obtained through differentiating (16) with respect to $P_{m}$ as

$$
\varphi+\chi P_{m}+\zeta P_{m}^{2}+\theta P_{m}^{3}+\vartheta P_{m}^{4}=0,
$$

where the values of $\varphi, \chi, \zeta, \theta$ and $\vartheta$ are given in (20)-(24) on the top of the next page. Note that, (19) is the polynomial of order four, the solution of which can be found easily through the conventional techniques or by using any polynomial solver. Since we aim to maximize the total energy efficiency of IoV network, thus, the value of $P_{m}^{*}$ is given by the larger root of (19). Substituting the optimal values of $\varpi_{s, m}^{*}, \varpi_{w, m}^{*}$ and $P_{m}^{*}$ , the problem (15) can be written as

$$
\begin{aligned}
& \underset{\left(\varpi_{m}^{*}, P_{m}^{*}\right)}{\operatorname{maximize}} \sum_{m=1}^{M}\left\{\log _{2}\left(1+\frac{P_{m}^{*} \varpi_{s, m}^{*} \mathcal{A}_{s, m}}{P_{m}^{*} \varpi_{w, m}^{*} \mathcal{B}_{s, m}+\mathcal{C}_{s, m}}\right)\right. \\
& \left.+\log _{2}\left(1+\frac{P_{m}^{*} \varpi_{w, m}^{*} \mathcal{A}_{w, m}}{P_{m}^{*} \varpi_{s, m}^{*} \mathcal{B}_{w, m}+\mathcal{C}_{w, m}}\right)\right\} \\
& -\beta \sum_{m=1}^{M} P_{m}^{*}\left(\varpi_{s, m}^{*}+\varpi_{w, m}^{*}\right)+P_{c},
\end{aligned}
$$

$$
\text { subject to: } \boldsymbol{\lambda}_{\boldsymbol{m}}, \mu_{m}, \epsilon_{m} \geq 0
$$

Finally, the dual variables $\lambda_{s, m}, \lambda_{w, m}, \mu_{m}$ and $\epsilon_{m}$ are iteratively updated as

$$
\begin{aligned}
\lambda_{s, m}(t+1) & =\lambda_{s, m}(t)+\delta(t)\left(P_{m}^{*} \varpi_{s, m}^{*} \mathcal{A}_{s, m}\right. \\
& \left.-\left(2^{R_{m i n}}-1\right) P_{m}^{*} \varpi_{s, m}^{*} \mathcal{B}_{s, m}+\mathcal{C}_{s, m}\right), \forall m \\
\lambda_{w, m}(t+1) & =\lambda_{w, m}(t)+\delta(t)\left(P_{m}^{*} \varpi_{w, m}^{*} \mathcal{A}_{w, m}\right. \\
& -\left(2^{R_{m i n}}-1\right)\left(P_{m}^{*} \varpi_{s, m}^{*} \mathcal{B}_{w, m}+\mathcal{C}_{w, m}\right), \forall m \\
\epsilon_{m}(t+1)= & \epsilon_{m}(t)+\delta(t)\left(1-\left(\varpi_{s, m}^{*}+\varpi_{w, m}^{*}\right)\right), \forall m, \\
\mu_{m}(t+1)= & \mu_{m}(t)+\delta(t)\left(P_{\max }-P_{m}^{*}\right), \forall m,
\end{aligned}
$$

where $t$ denotes the iteration index. The dual variables are iteratively updated until the stability criterion is reached.

\section{B. Efficient Reflection Power of BackTags}

Now we calculate the efficient reflection power of BackTags. For a given transmit power of RSUs, the optimization problem (P1) can be reformulated as

$$
\begin{gathered}
\text { (P3) } \underset{\left(\psi_{k, m}\right)}{\operatorname{maximize}} \sum_{m=1}^{M} R_{m}\left(\psi_{k, m}\right) \\
-\beta \sum_{m=1}^{M} P_{t o t}\left(\psi_{k, m}\right),
\end{gathered}
$$$$
\text { s.t. } \quad(7 a),(7 b),(7 d),(7 f) \text {, }
$$

Next we can rewrite (4) and (5) such as

$$
\begin{gathered}
\gamma_{s \rightarrow s}^{m}=\log _{2}\left(1+\frac{\mathcal{X}_{s, m}+\mathcal{Y}_{s, m}}{\mathcal{Z}_{s, m}}\right), \\
\gamma_{w \rightarrow w}^{m}=\log _{2}\left(1+\frac{\mathcal{X}_{w, m}+\mathcal{Y}_{w, m}}{\mathcal{Z}_{w, m}+\mathcal{W}_{w, m}}\right),
\end{gathered}
$$

where $\mathcal{X}_{s, m}=P_{m} \varpi_{s, m}^{*}\left|h_{s, m}\right|^{2}, \mathcal{Y}_{s, m}=P_{m} \varpi_{s, m}^{*} G_{s, m}$, $\mathcal{Z}_{s, m}=P_{m} \varpi_{w, m}^{*}\left|h_{s, m}\right|^{2} \Lambda+I_{s, m^{\prime}}^{k}+\sigma^{2}, \quad \mathcal{X}_{w, m}=$ $P_{m} \varpi_{w, m}^{*}\left|h_{w, m}\right|^{2}, \quad \mathcal{Y}_{w, m}=P_{m} \varpi_{w, m}^{*} G_{w, m}, \quad \mathcal{Z}_{w, m}=$ $P_{m} \varpi_{s, m}^{*}\left|h_{w, m}\right|^{2}+I_{w, m^{\prime}}^{m}+\sigma^{2}$ and $\mathcal{W}_{w, m}=P_{m} \varpi_{s, m}^{*} G_{w, m}$. 


$$
\begin{aligned}
\psi & =\mathcal{C}_{s, m} \mathcal{C}_{w, m}\left(-\mathcal{A}_{w, m} \mathcal{C}_{s, m}\left(-1+\varpi_{s, m}\right)\right)\left(1+\lambda_{w, m}\right)+\mathcal{C}_{w, m}\left(\mathcal{A}_{s, m} \varpi_{s, m}\left(1+\lambda_{s, m}\right)-\mathcal{C}_{s, m}\left(\mu_{m}+\beta\right)\right), \\
\chi & =\mathcal{C}_{s, m} \mathcal{C}_{w, m}\left(2\left(\mathcal{B}_{s, m} \mathcal{C}_{w, m}\left(-1+\varpi_{s, m}\right)-\mathcal{B}_{w, m} \mathcal{C}_{s, m} \varpi_{s, m}\right)\left(\mu_{m}+\beta\right)+\mathcal{A}_{w, m}\left(-1+\varpi_{s, m}\right)\left(2 \mathcal{B}_{s, m}\left(-1+\varpi_{s, m}\right)\left(1+\lambda_{w, m}\right)\right.\right. \\
& \left.-\mathcal{A}_{s, m} \varpi_{s, m}\left(2+\lambda_{s, m}+\lambda_{w, m}\right)+\mathcal{C}_{s, m}\left(\mu_{m}+\beta\right)\right)+\mathcal{A}_{s, m} \varpi_{s, m}\left(2 \mathcal{B}_{w, m} \varpi_{s, m}\left(1+\lambda_{s, m}\right)-\mathcal{C}_{w, m}\left(\mu_{m}+\beta\right)\right), \\
\zeta & =-\left(\mathcal{B}_{s, m}^{2} \mathcal{C}_{w, m}^{2}\left(-1+\varpi_{s, m}\right)^{2}-4 \mathcal{B}_{s, m} \mathcal{B}_{w, m} \mathcal{C}_{s, m} \mathcal{C}_{w, m}\left(-1+\varpi_{s, m}\right) \varpi_{s, m}+\mathcal{B}_{w, m}^{2} \mathcal{C}_{s, m}^{2} \varpi_{s, m}^{2}\right)\left(\mu_{m}+\beta\right)+\mathcal{A}_{s, m} \varpi_{s, m}\left(\mathcal{B}_{w, m}^{2}\right. \\
& \left.\mathcal{C}_{s, m} \varpi_{s, m}^{2}\left(1+\lambda_{s, m}\right)+\mathcal{B}_{s, m} \mathcal{C}_{w, m}^{2}\left(-1+\varpi_{s, m}\right)\left(\mu_{m}+\beta\right)-2 \mathcal{B}_{w, m} \mathcal{C}_{s, m} \mathcal{C}_{w, m} \varpi_{s, m}\left(\mu_{m}+\beta\right)\right)-\mathcal{A}_{w, m}\left(-1+\varpi_{s, m}\right) \\
& \left(\mathcal{B}_{s, m}^{2} \mathcal{C}_{w, m}\left(-1+\varpi_{s, m}\right)^{2}\left(1+\lambda_{w, m}\right)-\mathcal{B}_{s, m} \mathcal{C}_{w, m}\left(-1+\varpi_{s, m}\right)\left(\mathcal{A}_{s, m} \varpi_{s, m}\left(1+\lambda_{w, m}\right)-2 \mathcal{C}_{s, m}\left(\mu_{m}+\beta\right)\right)-\mathcal{C}_{s, m}\right. \\
& \left.\varpi_{s, m}\left(\mathcal{B}_{w, m} \mathcal{C}_{s, m}\left(\mu_{m}+\beta\right)+\mathcal{A}_{s, m}\left(-\mathcal{B}_{w, m} \varpi_{s, m}\left(1+\lambda_{s, m}\right)+\mathcal{C}_{w, m}\left(\mu_{m}+\beta\right)\right)\right)\right) \\
\theta & =\left(\mathcal { B } _ { w , m } \varpi _ { s , m } \left(-2 \mathcal{B}_{s, m}^{2} \mathcal{C}_{w, m}\left(-1+\varpi_{s, m}\right)^{2}+2 \mathcal{B}_{s, m}\left(\mathcal{B}_{w, m} \mathcal{C}_{s, m}+\mathcal{A}_{s, m} \mathcal{C}_{w, m}\right)\left(-1+\varpi_{s, m}\right) \varpi_{s, m}-\mathcal{A}_{s, m} \mathcal{B}_{w, m}\right.\right. \\
& \left.\mathcal{C}_{s, m} \varpi_{s, m}^{2}\right)+\mathcal{A}_{w, m}\left(-1+\varpi_{s, m}\right)\left(\mathcal{B}_{s, m}^{2} \mathcal{C}_{w, m}\left(-1+\varpi_{s, m}\right)^{2}-\mathcal{B}_{s, m}\left(2 \mathcal{B}_{w, m} \mathcal{C}_{s, m}+\mathcal{A}_{s, m} \mathcal{C}_{w, m}\right)\right. \\
& \left.\left(-1+\varpi_{s, m}\right) \varpi_{s, m}+\mathcal{A}_{s, m} \mathcal{B}_{w, m} \mathcal{C}_{s, m} \varpi_{s, m}^{2}\right)\left(\mu_{m}+\beta\right), \\
\vartheta & =-\mathcal{B}_{s, m} \mathcal{B}_{w, m}\left(-1+\varpi_{s, m}\right) \varpi_{s, m}\left(\mathcal{B}_{s, m}\left(-1+\varpi_{s, m}\right)-\mathcal{A}_{s, m} \varpi_{s, m}\right)\left(\mathcal{A}_{w, m}-\mathcal{A}_{w, m} \varpi_{s, m}+\mathcal{B}_{w, m} \varpi_{s, m}\right)\left(\mu_{m}+\beta\right) .
\end{aligned}
$$

Now we prove that the sum rate in (P3) is a concave function with respect to $\psi_{k, m}$.

\section{Proposition 2.}

$$
\begin{aligned}
& \log _{2}\left\{\left(1+\frac{\mathcal{X}_{s, m}+\mathcal{Y}_{s, m}}{\mathcal{Z}_{s, m}}\right)\right. \\
& \left.+\log _{2}\left(1+\frac{\mathcal{X}_{w, m}+\mathcal{Y}_{w, m}}{\mathcal{Z}_{w, m}+\mathcal{W}_{w, m}}\right)\right\},
\end{aligned}
$$

is a concave function with respect to $\Phi_{f, k}$.

Proof. Please see Appendix C.

Based on Proposition 2, problem (P3) is concave-convex, thus, we adopt KKT conditions for obtaining efficient reflection power at BackTags. After some straightforward steps, the closed-form expression can be derived as

$$
\psi_{k, m}=\left[\frac{\left(2^{R_{\min }}-1\right)-\mathcal{X}_{s, m}}{P_{m} \varpi_{s, m}^{*} G_{s, m}}\right],
$$

Proof. Please see Appendix D.

Now we discuss the complexity of the proposed resource allocation framework. It can be observed that the complexity of our optimization framework depends on the number of RSUs and their serving vehicles. Note that we calculate the complexity in terms of iterations required for convergence of different variables. Considering the number of RSUs is $M$ and the number of vehicles is $\Pi$. The complexity of the proposed framework in one iteration is $\mathcal{O}(M \Pi)$. We assume that the total number of iterations is $T$. Thus, the overall complexity of our optimization framework can be expressed as $\mathcal{O}(T M \Pi)$.

\section{NumericAl RESUlts}

To check the superiority of the proposed joint optimization framework, this section provides extensive results which are plotted over $10^{4}$ Monte Carlo simulations. We compare the proposed AmBC-enabled NOMA IoV framework with the conventional NOMA IoV framework without backscattering. Unless stated otherwise the network parameters are defined as: the channel is independent and identical Rayleigh fading. The power budget of each RSU is set as $P_{m}=18 \mathrm{dBm}$,

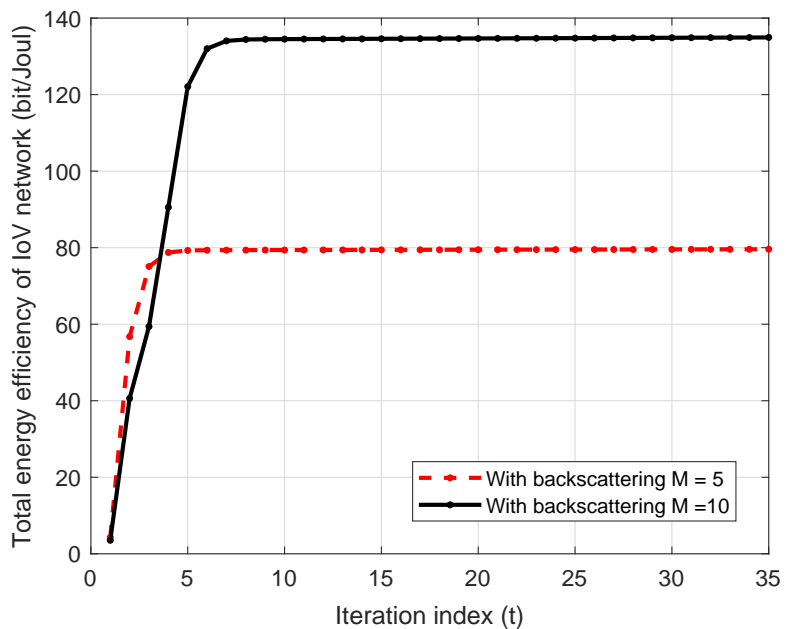

Fig. 2: Number of iterations versus the total energy efficiency of IoV network with different number of RSUs.

the variance of AWGN is $\sigma^{2}=0.1$, the number of RSU is $M=10$, the number of IoVs is set as $\Pi=20$, the imperfect SIC parameter is $\Lambda=0.1$ and 0.3 , the circuit power is set as $P_{c}=0.1$ and 0.5 .

The complexity in terms of iteration that requires for the convergence of the proposed IoV framework with backscattering is shown in Fig. 2. It can be seen that the total energy efficiency of the proposed IoV framework with backscattering converges after few iterations for different RSUs. We can also observe that when the number of RSUs in the network is increased, various optimization variables are also increased, which results in requiring more iterations for convergence. For example, the network with $M=5$ RSUs converges in five iterations, whereas the network having $M=10$ RSUs requires ten iterations for convergence. However, the proposed NOMA IoV framework with backscattering converges within a reasonable number of iterations.

To see the effect of RSU power budget and imperfect SIC parameter, Fig. 3 plots the total energy efficiency of the IoV network against the varying power of RSUs for different values 


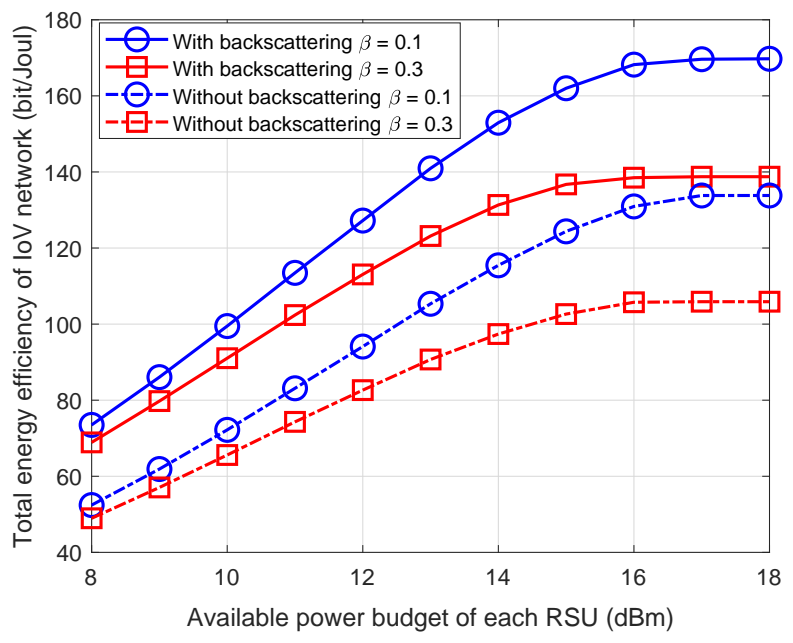

Fig. 3: Transmit power of SAPs versus the total achievable EE of SCNet for different values of imperfect SIC parameter.

of $\Lambda$. As the transmit power of RSUs increases, the total energy efficiency of the NOMA IoV framework with backscattering and without backscattering also increases. However, after a certain point, when the available power budget of RSUs further increases, the total energy efficiency of both IoV framework with backscattering and without backscattering remains unchanged. It is because at these points, the transmit power of RSUs is optimal, and assigning more transmit power would result in reducing the total energy efficiency of the IoV network. Thus, when the available power budget of RSUs further increases after these points, the transmit power of RSUs remains unchanged. Further, we can also observe that when $\Lambda=0.1$, the total energy efficiency is larger than that when $\Lambda=0.3$. This is because when $\beta$ has a small value, less interference is faced by the IoVs. However, increasing the value of $\Lambda$ results in more decoding error of signal which reduces the total energy efficiency. Overall, it is clear from the figure that the IoV framework with backscattering significantly outperforms the framework without backscattering for all values of the RSUs available power budget.

The total energy efficiency of the IoV network also depends on the number of RSUs in the system. Fig. 4 depicts the total energy efficiency of the IoV network against the increasing power budget of RSUs for different number of RSUs in the system. For both $M=5$ and 10 , the total energy efficiency of IoV framework with backscattering and without backscattering increases when the available power budget of RSUs increases. However, there is no further change in the total energy efficiency after a certain point because of the same reason as was discussed earlier. It is important to note that the performance gap between two IoV frameworks (with backscattering and without backscattering) increases when the available power budget of RSUs increases. This is because the high transmit power of RSUs also increases the data rate of BackTags which results in more energy efficiency compared to the IoV framework without backscattering. In addition, with more RSUs in the network, the advantage of IoV framework with backscattering becomes more clear compared to the one

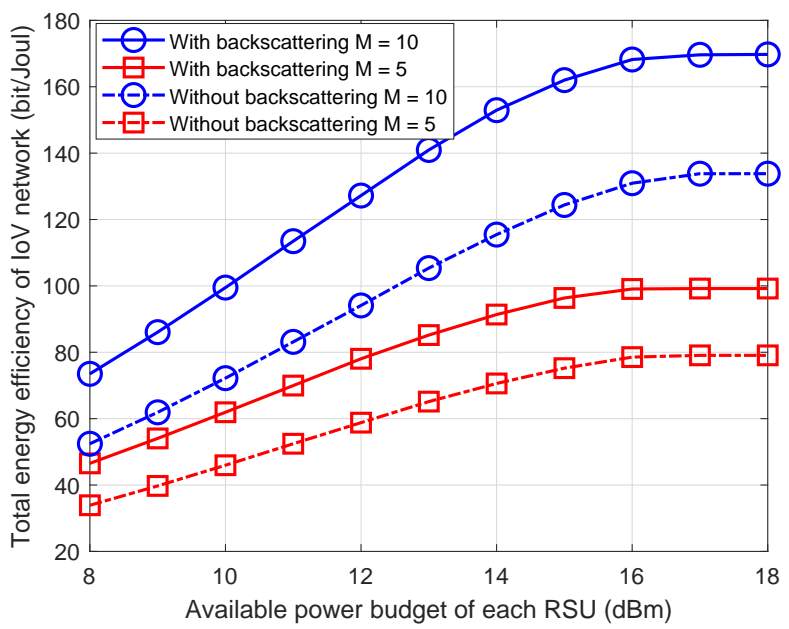

Fig. 4: Transmit power of SAPs versus the total achievable EE of SCNet for different number SAPs.

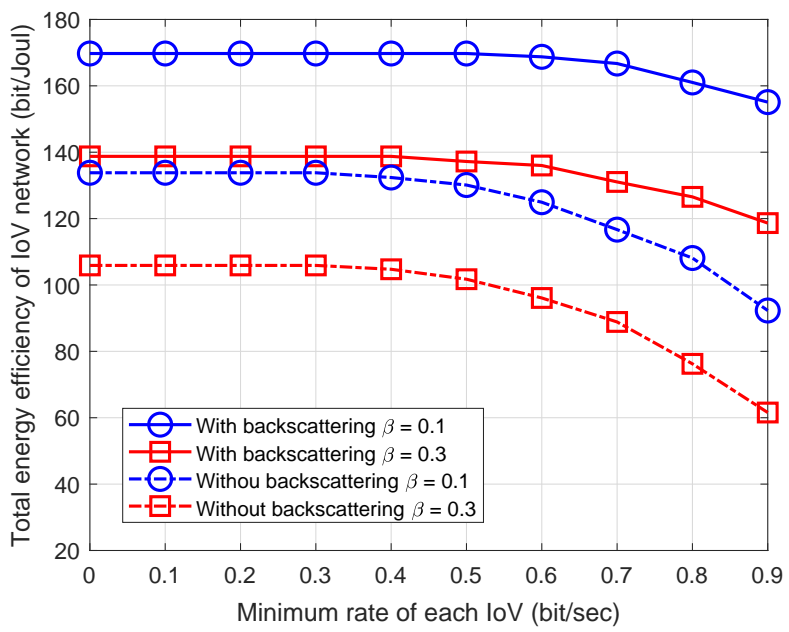

Fig. 5: QoS requirements versus the total achievable EE of SCNet for different values of imperfect SIC parameter.

without backscattering.

The impact of the minimum data rate of each IoV $\left(R_{\min }\right)$ in the network on the total energy efficiency of NOMA IoV network with backscattering and without backscattering is shown in Fig. 5. We can see that the total energy efficiency of the IoV network decreases as the minimum data rate of each $\mathrm{IoV}$ increases. This is because to achieve the minimum data rate of each IoV in the network, the transmit power of IoVs having comparatively bad channel conditions is increased. It results in the reduction of the total energy efficiency of the NOMA IoV network. If the minimum data rate cannot be achieved by changing the power allocation coefficient of IoVs, the system increases the power budget of RSUs which results in further reduction of the total energy efficiency of the IoV network. More specifically, the data rate is a logarithmic function of power, thus, when more transmit power is required to achieve the minimum data rate of IoVs, the total energy efficiency of the IoV network also reduces. This can be also proved from the definition of energy efficiency in (6). It is also 


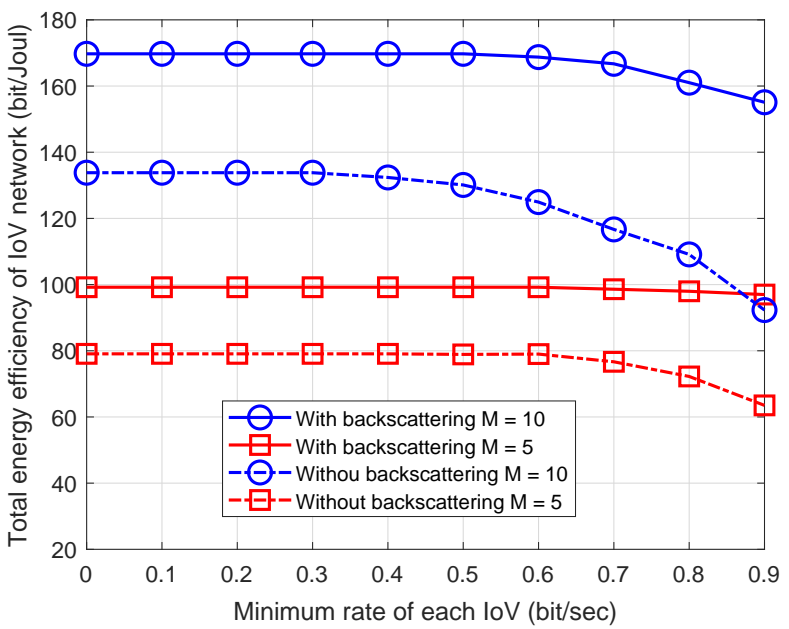

Fig. 6: QoS requirements versus the total achievable EE of SCNet for different number of SAPs.

interesting to see that when the minimum data rate of each IoV increases, the total energy efficiency of the IoV framework without backscattering decreases more rapidly compared to the IoV framework with backscattering. This is because when the total transmit power to achieve the minimum data rate is increased, the interference between different RSUs also increases which results in the reduction of the total energy efficiency of the IoV network. However, in the case of the IoV network with backscattering, the increase in the transmit power also increases the data rate of BackTags which further improves the total energy efficiency.

Fig. 6 shows that the performance gap between two frameworks increases as the number of total RSUs in the network increases. The main reason for this is because each geographical area has independent RSU, IoVs, and BackTag. Thus, when more number of RSUs and BackTags serve in the network, so the benefits of the IoV network with backscattering becomes more prominent. Another interesting thing to note is that when the minimum data rate is increased, the total energy efficiency of IoV network with backscattering and without backscattering decreases more rapidly in the case of $M=10$ RSUs compared to the system with $M=5$. This is because the IoVs in the network having more RSUs face more inter-RSU interference. Thus, as the transmit power increase to achieve the minimum data rate of IoVs, the total energy efficiency decreases rapidly, i.e., in the system with $M=10$, an IoV of one RSU faces interference from the remaining 9 RSUs. Again, for both $M=5$ and 10, the proposed IoV framework with backscattering significantly outperforms the benchmark IoV framework without backscattering.

Finally, it is important to show the effect of the circuit power on the total energy efficiency of both IoV frameworks with backscattering and without backscattering. It can be seen from Fig. 7 that the high value of circuit power decreases the total energy efficiency of the IoV network. However, the proposed IoV framework with backscattering achieves high energy efficiency than without a backscattering framework. One should be also noted from the figure that when the circuit

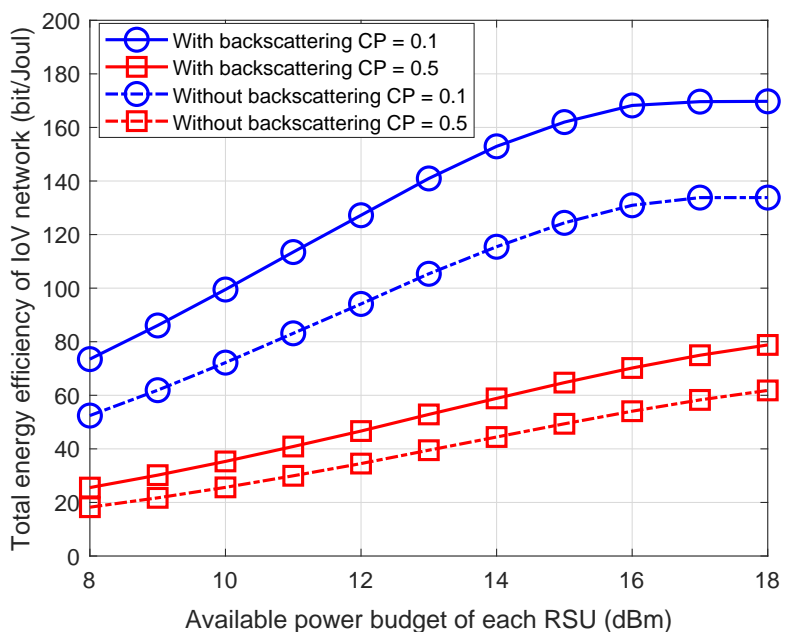

Fig. 7: QoS requirements versus the total achievable EE of SCNet for different number of SAPs.

power is $P_{c}=5$, the optimal transmit power of RSUs is achieved at comparatively larger values of the power budget. Moreover, when the available power budget of RSUs increases, the gap of total energy efficiency between both frameworks also increases. This is because the proposed IoV framework with backscattering is more efficient than the one without backscattering.

\section{CONCLUSIONS}

This paper has provided a green optimization framework for AmBC-enabled NOMA vehicular networks under imperfect SIC decoding. In particular, the transmit power of RSUs and reflection power of BackTags are simultaneously optimized to maximize the total achievable energy efficiency of the network. Dinkelbach method has been exploited first to transform the objective function of the non-convex problem into subtractive function. The transformed problem has then been decoupled into two subproblems for transmitting power allocation at RSUs and reflection power at BackTags. Closed-form solutions have been explored through dual theory and KKT conditions. Simulation results demonstrate that the proposed optimization framework with backscattering outperforms the benchmark optimization framework without backscattering.

This work can be extended in several ways. For example, we can investigate the total energy efficiency of the same system under the vehicle's mobility and imperfect channel state information. The same system can also be extended to multi-carrier communication such that each RSU will accommodate multiple vehicles at one time. Besides that, we can also consider multiple BackTags in each RSU. In such a scenario, we can select a BackTag which provides a high data rate. These interesting works will be explored in our future studies.

\section{ApPendix A: Proof Of Proposition 1}

The concavity proof of the objective function of (P2) with respect to $\varpi_{s, m}$ and $\Lambda_{w, m}$ is discussed here. It is important to mention that a function should be concave if its Hessian matrix 
is negative definite. For instance, a matrix is negative definite if its principal minors are alternate $i$ sign. Based on the above observations, we now define and derive the Hessian matrix of (P2). Subsequently, we will prove it as negative definite. Since the second part of the objective function of (P2), i.e., $-\beta \sum_{m=1}^{M} P_{m}\left(\varpi_{s, m}+\varpi_{w, m}\right)+P_{c}$ is a linear function of $\varpi_{s, m}$ and $\varpi_{w, m}$, its first part can be written as:

$$
\begin{aligned}
& \sum_{m=1}^{M}\left\{\log _{2}\left(1+\frac{P_{m} \varpi_{s, m} \mathcal{A}_{s, m}}{P_{m} \varpi_{w, m} \mathcal{B}_{s, m}+\mathcal{C}_{s, m}}\right)\right. \\
& \left.+\log _{2}\left(1+\frac{P_{m} \varpi_{w, m} \mathcal{A}_{w, m}}{P_{m} \varpi_{s, m} \mathcal{B}_{w, m}+\mathcal{C}_{w, m}}\right)\right\},
\end{aligned}
$$

Next, we derive the Hessian matrix of (A1) as:

$$
H=\left[\begin{array}{cc}
\frac{\partial(\mathrm{A} 1)}{\partial^{2} \varpi_{s, m}} & \frac{\partial(\mathrm{A} 1)}{\partial \varpi_{s, m} \partial \varpi_{w, m}} \\
\frac{\partial(\mathrm{A} 1)}{\partial \varpi_{w, m} \partial \varpi_{s, m}} & \frac{\partial(\mathrm{A} 1)}{\partial^{2} \varpi_{s, m}}
\end{array}\right]
$$

$$
\begin{aligned}
& \frac{\partial(\mathrm{A} 1)}{\partial^{2} \varpi_{s, m}}=\Theta_{1,1}=- \\
& \frac{\mathcal{A}_{s, m}^{2} \mathcal{V}_{w, m}^{2} \mathcal{T}_{w, m}^{2}-\mathcal{A}_{w, m} \mathcal{B}_{w, m}^{2} \mathcal{T}_{s, m}^{2}\left(2 \mathcal{V}_{w, m}+\mathcal{A}_{w, m} \varpi_{w, m}\right) \varpi_{w, m}}{\ln (2) \mathcal{T}_{s, m}^{2} \mathcal{T}_{w, m}^{2} \mathcal{V}_{w, m}^{2}}
\end{aligned}
$$

$$
\begin{aligned}
& \frac{\partial(\mathrm{A} 1)}{\partial \varpi_{s, m} \partial \varpi_{w, m}}=\Theta_{1,2} \\
& =-\frac{\mathcal{A}_{s, m} \mathcal{B}_{s, m} \mathcal{T}_{w, m}^{2}-\mathcal{A}_{w, m} \mathcal{B}_{w, m} \mathcal{T}_{s, m}^{2}}{\ln (2) \mathcal{T}_{s, m}^{2} \mathcal{T}_{w, m}^{2}}
\end{aligned}
$$

$$
\begin{aligned}
& \frac{\partial(\mathrm{A} 1)}{\partial^{2} \varpi_{w, m}}=\varphi_{2,1}=- \\
& \frac{\mathcal{A}_{w, m}^{2} \mathcal{V}_{s, m}^{2} \mathcal{T}_{s, m}^{2}-\mathcal{A}_{s, m} \mathcal{B}_{s, m}^{2} \mathcal{T}_{w, m}^{2}\left(2 \mathcal{V}_{s, m}+\mathcal{A}_{s, m} \varpi_{s, m}\right) \varpi_{s, m}}{\ln (2) \mathcal{T}_{w, m}^{2} \mathcal{T}_{s, m}^{2} \mathcal{V}_{s, m}^{2}}
\end{aligned}
$$

$$
\begin{aligned}
& \frac{\partial(\mathrm{A} 1)}{\partial \varpi_{w, m} \partial \varpi_{s, m}}=\Theta_{2,2} \\
& =-\frac{\mathcal{A}_{s, m} \mathcal{B}_{s, m} \mathcal{T}_{w, m}^{2}-\mathcal{A}_{w, m} \mathcal{B}_{w, m} \mathcal{T}_{s, m}^{2}}{\ln (2) \mathcal{T}_{s, m}^{2} \mathcal{T}_{w, m}^{2}}
\end{aligned}
$$

where, $\mathcal{T}_{s, m}=\mathcal{A}_{s, m} \varpi_{s, m}+\mathcal{V}_{s, m}, \mathcal{T}_{w, m}=\mathcal{A}_{w, m} \varpi_{w, m}+$ $\mathcal{V}_{w, m}, \mathcal{V}_{s, m}=\mathcal{B}_{s, m} \varpi_{w, m}+\mathcal{C}_{s, m}$, and $\mathcal{V}_{w, m}=\mathcal{B}_{w, m} \varpi_{s, m}+$ $\mathcal{C}_{w, m}$. The obtained Hessian matrix can be expressed as:

$$
H=\left[\begin{array}{ll}
\Theta_{1,1} & \Theta_{1,2} \\
\Theta_{2,1} & \Theta_{2,2}
\end{array}\right]
$$

The first order principal minors of the obtained Hessian matrix are $\Theta_{1,1}$ and $\Theta_{2,2}$ which are negative. The second-order principal minor of the $\mathrm{H}$ is the determinant of the $\mathrm{H}$.

$$
\operatorname{det} H=\Theta_{1,1} \Theta_{2,2}-\Theta_{1,2} \Theta_{2,1}>0
$$

Hence, Proposition 1 is proved.

\section{APPENDIX B: PROOF OF PROPOSITION 2}

To prove the closed-form solution in (17), we calculate partial derivation of (16) with respect to $\varpi_{s, m}$ as

$$
\begin{aligned}
& \frac{\partial L\left(\varpi_{\boldsymbol{m}}, \boldsymbol{\lambda}_{\boldsymbol{m}}, \mu_{m}, \epsilon_{m}\right)}{\partial \varpi_{s, m}}= \\
& \frac{\mathcal{A}_{s, m}}{\ln (2)\left(\mathcal{A}_{s, m} \varpi_{s, m}+\mathcal{B}_{s, m} \varpi_{w, m}+\mathcal{C}_{s, m}\right)}-\mathcal{D}+ \\
& \frac{\mathcal{A}_{w, m} \mathcal{B}_{w, m} \varpi_{w, m}}{\ln (2)\left(\mathcal{B}_{w, m} \varpi_{s, m}+\mathcal{C}_{w, m}\right)\left(\mathcal{A}_{w, m-} \varpi_{w, m}+\mathcal{B}_{w, m} \varpi_{s, m}+\mathcal{C}_{w, m}\right)}
\end{aligned}
$$

where $\mathcal{D}=\beta P_{m}-\lambda_{s, m} \mathcal{A}_{s, m}+\lambda_{w, m}\left(2^{R_{m i n}}-1\right) \mathcal{B}_{w, m}+\epsilon_{m}$. Now set $\varpi_{w, m}=1-\varpi_{s, m}$ in (B1), it can be stated as

$\frac{\mathcal{A}_{s, m}}{\ln (2)\left(\mathcal{X}_{s, m} \varpi_{s, m}+\mathcal{W}_{s, m}\right)}-\frac{\gamma_{w \rightarrow w}^{m} \mathcal{B}_{w, m}}{\ln (2)\left(\mathcal{Y}_{w, m} \varpi_{s, m}+\mathcal{W}_{w, m}\right)}-\mathcal{D}$

where $\mathcal{X}_{s, m}=\mathcal{A}_{s, m}-\mathcal{B}_{s, m}, \mathcal{Y}_{w, m}=\mathcal{B}_{w, m}-\mathcal{A}_{w, m}, \mathcal{W}_{s, m}=$ $\mathcal{B}_{s, m}+\mathcal{C}_{s, m}$ and $\mathcal{W}_{w, m}=\mathcal{A}_{w, m}+\mathcal{C}_{w, m}$. After straightforward calculation, it can be written as

$\mathcal{A}_{s, m}\left(\mathcal{Y}_{w, m} \varpi_{s, m}+\mathcal{W}_{w, m}\right)-\gamma_{w \rightarrow w}^{m} \mathcal{B}_{w, m}\left(\mathcal{X}_{s, m} \varpi_{s, m}+\mathcal{W}_{s, m}\right)$

$-\ln (2) \mathcal{D}\left(\mathcal{Y}_{w, m} \varpi_{s, m}+\mathcal{W}_{w, m}\right)\left(\mathcal{X}_{s, m} \varpi_{s, m}+\mathcal{W}_{s, m}\right)=0$

By using quadratic formula $a x^{2}+b x+c$, it can be expressed as

$$
\begin{aligned}
& \left(-\ln (2) \mathcal{D} \mathcal{X}_{s, m} \mathcal{Y}_{w, m}\right) \varpi_{s, m}^{2}+\left(\mathcal{A}_{s, m} \mathcal{Y}_{w, m}-\gamma_{w \rightarrow w}^{m} \mathcal{B}_{w, m} \mathcal{X}_{s, m}\right. \\
& \left.-\ln (2) \mathcal{D} \mathcal{X}_{s, m} \mathcal{W}_{w, m}-\ln (2) \mathcal{D} \mathcal{Y}_{w, m} \mathcal{W}_{s, m}\right) \varpi_{s, m}+ \\
& \left(\mathcal{A}_{s, m} \mathcal{W}_{w, m}-\gamma_{w \rightarrow w}^{m} \mathcal{B}_{w, m} \mathcal{W}_{s, m}-\ln (2) \mathcal{D} \mathcal{W}_{s, m} \mathcal{W}_{w, m}\right)
\end{aligned}
$$

Now solving for $\varpi_{s, m}$, it results as

$$
\varpi_{s, m}=\left[\frac{-\Psi \pm \sqrt{\Psi^{2}-4 \Phi \Upsilon}}{2 \Phi}\right]^{+}
$$

Thus, Proposition 2 is proved.

\section{Appendix C: Proof Of Proposition 3}

Here, we prove the concavity of (33) with respect to $\psi_{k, m}$. To do so, we calculate first and second-order derivatives of (33) with respect to $\psi_{k, m}$. The first order derive can be computed as

$$
\begin{aligned}
\frac{\partial}{\partial \psi_{k, m}} & {\left[\operatorname { l o g } _ { 2 } \left\{\left(1+\frac{\mathcal{X}_{s, m}+\mathcal{Y}_{s, m}}{\mathcal{Z}_{s, m}}\right)\right.\right.} \\
& \left.\left.+\log _{2}\left(1+\frac{\mathcal{X}_{w, m}+\mathcal{Y}_{w, m}}{\mathcal{Z}_{w, m}+\mathcal{W}_{w, m}}\right)\right\}\right],
\end{aligned}
$$

After calculating the partial derivative, it can be expressed as

$$
\frac{\mathcal{Y}_{s, m}}{\ln (2)\left(\mathcal{A}_{s, m}+\mathcal{Z}_{s, m}\right)}+\frac{\mathcal{C}_{w, m}}{\ln (2)\left(\mathcal{B}_{w, m}{ }^{2}+\mathcal{B}_{w, m} \mathcal{A}_{w, m}\right)}
$$

where $\mathcal{A}_{s, m}=\left(\mathcal{X}_{s, m}+\psi_{k, m} \mathcal{Y}_{s, m}\right), \mathcal{A}_{w, m}=\left(\mathcal{X}_{w, m}+\right.$ $\left.\psi_{k, m} \mathcal{Y}_{w, m}\right), \mathcal{B}_{w, m}=\left(\mathcal{Z}_{w, m}+\psi_{k, m} \mathcal{W}_{w, m}\right)$, and $\mathcal{C}_{w, m}=$ 
$\left(\mathcal{Y}_{w, m} \mathcal{Z}_{w, m}-\mathcal{X}_{w, m} \mathcal{W}_{w, m}\right)$. Next we calculate its second derivative as

$$
\frac{\partial^{2}}{\partial \psi_{k, m}^{2}}\left(\frac{\mathcal{Y}_{s, m}}{\ln (2)\left(\mathcal{A}_{s, m}+\mathcal{Z}_{s, m}\right)}+\frac{\mathcal{C}_{w, m}}{\ln (2)\left(\mathcal{B}_{w, m}{ }^{2}+\mathcal{B}_{w, m} \mathcal{A}_{w, m}\right)}\right)
$$

After deriving (C3), it can be stated as

$$
\begin{aligned}
& -\left(\frac{\mathcal{Y}_{s, m}{ }^{2}}{\ln (2)\left(\mathcal{A}_{s, m}+\mathcal{Z}_{s, m}\right)^{2}}\right. \\
& \left.+\frac{\mathcal{C}_{w, m}\left(2 \mathcal{W}_{w, m} \mathcal{E}_{w, m}+\mathcal{C}_{w, m}^{+}\right)}{\ln (2) \mathcal{B}_{w, m}^{2}\left(\mathcal{B}_{w, m}+\mathcal{B}_{w, m} \mathcal{A}_{w, m}\right)^{2}}\right)<0
\end{aligned}
$$

where $\mathcal{E}_{w, m}=\mathcal{B}_{w, m}+\mathcal{Y}_{w, m} \psi_{k, m}$, and $\mathcal{C}_{w, m}^{+}=$ $\left(\mathcal{Y}_{w, m} \mathcal{Z}_{w, m}+\mathcal{X}_{w, m} \mathcal{W}_{w, m}\right)$.

It can be observed that the second order derivative of (33) is less than zero, hence it is a concave and increasing function of $\psi_{k, m}$.

\section{APPENDix D: Proof OF Proposition 4}

To obtain the closed-form solution in (34), we adopt KKT conditions. To do so, we first define the Lagrangian function of problem (P3) such as

$$
\begin{aligned}
& \mathcal{L}\left(\psi_{k, m}, \lambda_{s, m}, \lambda_{w, m}, \mu_{m}, \eta_{k, m}\right)= \\
& \log _{2}\left\{\left(1+\frac{\mathcal{X}_{s, m}+\mathcal{Y}_{s, m}}{\mathcal{Z}_{s, m}}\right)+\log _{2}\left(1+\frac{\mathcal{X}_{w, m}+\mathcal{Y}_{w, m}}{Z_{w, m}+\mathcal{W}_{w, m}}\right)\right\} \\
& -\beta \sum_{m=1}^{M} P_{m}\left(\varpi_{s, m}+\varpi_{w, m}\right)+P_{c}+\lambda_{s, m}\left(\mathcal{X}_{s, m}+\mathcal{Y}_{s, m}-\right. \\
& \left.\left(2^{\mathcal{R}_{m i n}}-1\right) \mathcal{Z}_{s, m}\right)+\lambda_{w, m}\left(\mathcal{X}_{w, m}+\mathcal{Y}_{w, m}-\left(2^{R_{m i n}}-1\right) \times\right. \\
& \left.\left(\mathcal{Z}_{w, m}+\mathcal{W}_{w, m}\right)\right)+\mu_{m}\left(P_{\text {max }}-P_{m}\right)+\eta_{k, m}\left(\psi_{k, m}-1\right)
\end{aligned}
$$

where $\lambda_{s, m}, \lambda_{w, m}, \mu_{m}$ and $\eta_{k, m}$ are the Lagrangian multipliers. Next, we calculate the partial derivative of (D1) with respect to $\psi_{k, m}$ and set it equal to zero. By doing this, it results as

$$
\begin{aligned}
& \frac{\mathcal{Y}_{s, m}}{\ln (2)\left(\mathcal{A}_{s, m}+\mathcal{Z}_{s, m}\right)}+\frac{\mathcal{C}_{w, m}}{\ln (2)\left(\mathcal{B}_{w, m}^{2}+\mathcal{B}_{w, m} \mathcal{A}_{w, m}\right)}+\lambda_{s, m} \\
& Y_{s, m}+\lambda_{w, m}\left(\mathcal{Y}_{w, m}-\left(2^{\mathcal{R}_{m i n}}-1\right) \mathcal{W}_{w, m}\right)+\eta_{k, m}=0 \text { (D2) } \\
& \frac{\mathcal{Y}_{s, m}}{\ln (2)\left(\mathcal{A}_{s, m}+\mathcal{Z}_{s, m}\right)}+\frac{\mathcal{C}_{w, m}}{\ln (2)\left(\mathcal{B}_{w, m}^{2}+\mathcal{B}_{w, m} \mathcal{A}_{w, m}\right)}+\eta_{k, m} \\
& =\left(\lambda_{w, m}\left(2^{R_{m i n}}-1\right) \mathcal{W}_{w, m}-\lambda_{w, m} \mathcal{Y}_{w, m}\right)-\lambda_{s, m} \mathcal{Y}_{s, m}
\end{aligned}
$$

where $\mathcal{C}_{w, m}=\mathcal{Y}_{w, m} \mathcal{Z}_{w, m}-\mathcal{X}_{w, m} \mathcal{W}_{w, m}=I_{w, m^{\prime}}^{m}+\sigma^{2}>0$. It can be observed that the left side of (D3) always gives positive value, thus the right side value can stated as

$$
\left(\lambda_{w, m}\left(2^{R_{m i n}}-1\right) \mathcal{W}_{w, m}-\lambda_{w, m} \mathcal{Y}_{w, m}\right)>\lambda_{s, m} \mathcal{Y}_{s, m}
$$

where $\left(\lambda_{w, m}\left(2^{R_{\min }}-1\right) \mathcal{W}_{w, m}-\lambda_{w, m} \mathcal{Y}_{w, m}\right)$ is always positive because $\left(2^{R_{\text {min }}}-1\right)$ is always positive and $\mathcal{W}_{w, m}>$ $\mathcal{Y}_{w, m}$. Since $\lambda_{s, m} \geq 0$, the $\lambda_{w, m}$ is always positive. Thus, slack complimentary condition of KKT method should be satisfied. Moreover, constraints associated to $\lambda_{s, m}$ and $\lambda_{w, m}$ are also active. Hence, both constraints are equal to zero. Finally, efficient $\psi_{k, m}$ can be obtained through active inequality constraint as provided in (34).

\section{REFERENCES}

[1] W. U. Khan et al., "NOMA-enabled optimization framework for nextgeneration small-cell IoV networks under imperfect SIC decoding," IEEE Trans. Intell. Transp. Syst., pp. 1-1, 2021.

[2] A. Lamssaggad et al., "A survey on the current security landscape of intelligent transportation systems," IEEE Access, vol. 9, pp. 9180-9208, 2021.

[3] F. Jameel et al., "Efficient power-splitting and resource allocation for cellular V2X communications," IEEE Trans. Intell. Transp. Syst., pp. $1-10,2020$.

[4] C. De Alwis et al., "Survey on 6G frontiers: Trends, applications, requirements, technologies and future research," IEEE Open J. Commun. Society, pp. 1-1, 2021.

[5] U. M. Malik, M. A. Javed, S. Zeadally, and S. u. Islam, "Energy efficient fog computing for $6 \mathrm{G}$ enabled massive IoT: Recent trends and future opportunities," IEEE Internet of Things Journal, 2021, "to appear".

[6] Y. Hui et al., "Secure and personalized edge computing services in 6G heterogeneous vehicular networks," IEEE Internet Things J., pp. 1-1, 2021.

[7] Q. Wu et al., "Intelligent reflecting surface aided wireless communications: A tutorial," IEEE Trans. Commun., pp. 1-1, 2021.

[8] S. Tripathi et al., "Millimeter-wave and terahertz spectrum for $6 \mathrm{G}$ wireless," arXiv preprint arXiv:2102.10267, 2021.

[9] M. Kouhizadeh et al., "Blockchain technology and the sustainable supply chain: Theoretically exploring adoption barriers," Int. J. Production Economics, vol. 231, p. 107831, 2021.

[10] F. Jameel et al., "NOMA-enabled backscatter communications: Toward battery-free IoT networks," IEEE Internet Things Mag., vol. 3, no. 4, pp. 95-101, 2020.

[11] W. U. Khan et al., "Spectral efficiency optimization for next generation NOMA-enabled IoT networks," IEEE Trans. Veh. Technol., vol. 69, no. 12 , pp. $15284-15297$, Dec. 2020.

[12] M. A. Javed and S. Zeadally, "AI-Empowered Mobile Edge Computing in the Internet of Vehicles," IEEE Network, 2021, "to appear".

[13] X. Li et al., "Physical layer security of cognitive ambient backscatter communications for green Internet-of-things," IEEE Trans. Green Commun. Netw., pp. 1-1, 2021.

[14] W. U. Khan et al., "Joint spectral and energy efficiency optimization for downlink NOMA networks," IEEE Trans. Cogn. Commun. Netw., vol. 6, no. 2, pp. 645-656, 2020

[15] W. U. Khan, F. Jameel, X. Li, M. Bilal, and T. Tsiftsis, "Joint spectrum and energy optimization of NOMA-enabled small-cell networks with QoS guarantee," IEEE Trans. Veh. Technol., 2021.

[16] Z. Ali et al., "Fair power allocation in cooperative cognitive systems under NOMA transmission for future IoT networks," 2021.

[17] W. U. Khan et al., "Energy efficiency maximization for beyond 5G NOMA-enabled heterogeneous networks," Peer-to-Peer Networking and Applications, pp. 1-15, 2021.

[18] S. Yu et al., "Optimal power allocation for NOMA-enabled D2D communication with imperfect SIC decoding," Physical Communication, p. 101296, 2021.

[19] Z. Ali et al., "Artificial intelligence techniques for rate maximization in interference channels," Physical Communication, vol. 47, p. 101294, 2021.

[20] B. Wang et al., "Interference hypergraph-based 3D matching resource allocation protocol for NOMA-V2X networks," IEEE Access, vol. 7, pp. 90 789-90 800, 2019.

[21] O. Abbasi et al., "Trajectory design and power allocation for droneassisted NR-V2X network with dynamic NOMA/OMA," IEEE Trans Wireless Commun., vol. 19, no. 11, pp. 7153-7168, Nov. 2020.

[22] H. Zheng et al., "Joint resource allocation with weighted max-min fairness for NOMA-enabled V2X communications," IEEE Access, vol. 6, pp. 65 449-65 462, 2018.

[23] D. Zhang et al., "Performance analysis of FD-NOMA-based decentralized V2X systems," IEEE Trans. Commun., vol. 67, no. 7, pp. 50245036, July 2019.

[24] H. Xiao et al., "Power control for clustering car-following V2X communication system with non-orthogonal multiple access," IEEE Access, vol. 7, pp. $68160-68171,2019$. 
[25] B. Di et al., "Non-Orthogonal Multiple Access for High-Reliable and Low-Latency V2X Communications in 5G Systems," IEEE J. Sel. Areas Commun., vol. 35, no. 10, pp. 2383-2397, Oct. 2017.

[26] S. Guo et al., "Robust resource allocation with imperfect channel estimation in NOMA-based heterogeneous vehicular networks," IEEE Trans. Commun., vol. 67, no. 3, pp. 2321-2332, Mar. 2019.

[27] W. U. Khan et al., "Multiobjective optimization of uplink NOMAenabled Vehicle-to-Infrastructure communication," IEEE Access, vol. 8, pp. 84467-84 478, 2020.

[28] A. Ihsan et al., "Energy-efficient NOMA multicasting system for 5G cellular V2X communications with imperfect CSI," arXiv preprint arXiv:2009.03507, 2020.

[29] D.-T. Do et al., "Joint full-duplex and roadside unit selection for NOMAenabled V2X communications: Ergodic rate performance," IEEE Access, vol. 8, pp. 140348-140360, 2020.

[30] Q. Zhang et al., "Backscatter-NOMA: A symbiotic system of cellular and Internet-of-Things networks," IEEE Access, vol. 7, pp. 20000 20013, 2019.

[31] W. U. Khan et al., "NOMA-enabled wireless powered backscatter communications for secure and green IoT networks," in Wireless-Powered Backscatter Communications for Internet of Things. Springer, 2021, pp. 103-131.

[32] — "Secure backscatter communications in multi-cell NOMA networks: Enabling link security for massive IoT networks," in IEEE INFOCOM 2020-IEEE Conference on Computer Communications Workshops (INFOCOM WKSHPS). IEEE, 2020, pp. 213-218.

[33] X. Li et al., "Secrecy analysis of ambient backscatter NOMA systems under I/Q imbalance," IEEE Trans. Veh. Technol., vol. 69, no. 10, pp. $12286-12290$, Oct. 2020.

[34] _ - "Hardware impaired ambient backscatter NOMA systems: Reliability and security," IEEE Trans. Commun., pp. 1-1, 2021.

[35] J. Guo et al., "Design of non-orthogonal multiple access enhanced backscatter communication," IEEE Trans. Wireless Commun., vol. 17, no. 10, pp. 6837-6852, Oct. 2018.

[36] W. U. Khan et al., "Joint optimization of NOMA-enabled backscatter communications for beyond 5G IoT networks," Internet Technol. Lett., p. e265.

[37] _ , "Backscatter-enabled NOMA for future 6G systems: A new optimization framework under imperfect SIC," IEEE Commun. Lett., pp. 1-, 2021.

[38] Y. Liao et al., "Resource allocation in NOMA-enhanced full-duplex symbiotic radio networks," IEEE Access, vol. 8, pp. 22 709-22 720, 2020.

[39] G. Yang et al., "Resource allocation in NOMA-enhanced backscatter communication networks for wireless powered IoT," IEEE Wireless Commun. Lett., vol. 9, no. 1, pp. 117-120, 2019.

[40] Y. Xu et al., "Energy efficiency maximization in NOMA enabled backscatter communications with QoS guarantee," IEEE Wireless Commun. Lett., vol. 10, no. 2, pp. 353-357, Feb. 2021.

[41] F. Jameel et al., "Towards intelligent IoT networks: Reinforcement learning for reliable backscatter communications," in 2019 IEEE Globecom Workshops (GC Wkshps). IEEE, 2019, pp. 1-6.

[42] _ "Reinforcement learning for scalable and reliable power allocation in SDN-based backscatter heterogeneous network," in IEEE INFOCOM 2020-IEEE Conference on Computer Communications Workshops (INFOCOM WKSHPS). IEEE, 2020, pp. 1069-1074.

[43] W. U. Khan, Z. Ali, A. U. Khan, and G. A. S. Sidhu, "Secure backscatter-enabled NOMA system design in 6G era," Internet Technology Letters, p. e307.

[44] W. U. Khan et al., "Backscatter-enabled efficient V2X communication with non-orthogonal multiple access," IEEE Trans. Veh. Technol., vol. 70, no. 2, pp. 1724-1735, Feb. 2021.

[45] _ - "Efficient power allocation in downlink multi-cell multi-user NOMA networks," IET Commun., vol. 13, no. 4, pp. 396-402, 2019.

[46] Y. Liu et al., "Energy-efficient subchannel matching and power allocation in NOMA autonomous driving vehicular networks," IEEE Wireless Commun., vol. 26, no. 4, pp. 88-93, Aug. 2019. 